\title{
Anticancer and apoptotic activities of oleanolic acid are mediated through cell cycle arrest and disruption of mitochondrial membrane potential in HepG2 human hepatocellular carcinoma cells
}

\author{
YUE-YONG ZHU ${ }^{1}$, HONG-YAN HUANG ${ }^{2}$ and YIN-LIAN WU ${ }^{1}$ \\ ${ }^{1}$ Liver Center, The First Affiliated Hospital of Fujian Medical University, Fuzhou, Fujian 350005; \\ ${ }^{2}$ Department of Gastroenterology, Taizhou Municipal Hospital, Taizhou, Zhejiang 318000, P.R. China
}

Received May 26, 2014; Accepted March 12, 2015

DOI: $10.3892 / \mathrm{mmr} .2015 .4033$

\begin{abstract}
Hepatocellular carcinoma (HCC) is an aggressive form of cancer, with high rates of morbidity and mortality, a poor prognosis and limited therapeutic options. The objective of the present study was to demonstrate the anticancer activity of oleanolic acid in HepG2 human HCC cells. Cell viability was evaluated using an MTT assay, following administration of various doses of oleanolic acid. The effect of oleanolic acid on cell cycle phase distribution and mitochondrial membrane potential was evaluated using flow cytometry with propidium iodide and rhodamine-123 DNA-binding cationic fluorescent dyes. Fluorescence microscopy was employed to detect morphological changes in HepG2 cells following oleanolic acid treatment. The results revealed that oleanolic acid induced a dose-dependent, as well as time-dependent inhibition in the growth of HepG2 cancer cells. Following acridine orange and ethidium bromide staining, treatment with various doses $(0,5,25$ and $50 \mu \mathrm{M})$ of oleanolic acid induced typical morphological changes associated with apoptosis, including cell shrinkage, membrane blebbing, nuclear condensation and apoptotic body formation. Cell cycle analysis revealed that oleanolic acid induced cell cycle arrest in HepG2 cells at the sub-G1 (apoptotic) phase of the cell cycle, in a dose-dependent manner. Staining with Annexin V-fluorescein isothiocyanate and propidium iodide revealed that apoptosis occurred early in these cells. Oleanolic acid treatment also resulted in fragmentation of nuclear DNA in a dose-dependent manner, producing the typical features of DNA laddering on an agarose gel. The results also demonstrated that oleanolic acid treatment resulted in a potent loss of mitochondrial membrane potential,
\end{abstract}

Correspondence to: Dr Yue-Yong Zhu, Liver Center, The First Affiliated Hospital of Fujian Medical University, 20 Chazhong Road, Fuzhou, Fujian 350005, P.R. China

E-mail: zhuyueyong2002@gmail.com

Key words: hepatocellular carcinoma, oleanolic acid, apoptosis, flow cytometry, apoptotic bodies which also occurred in a dose-dependent manner. Therefore, oleanolic acid may be used as a therapeutic agent in the treatment of human HCC.

\section{Introduction}

In humans, aggressive types of cancer are the second leading cause of mortality (1). Among malignant tumors, hepatocellular carcinoma (HCC) is one of the most severe, with high morbidity and mortality rates, and a poor prognosis (2). There is an increasing incidence of HCC in China, where it accounts for $90 \%$ of cases of primary liver cancer, meaning that HCC is the second most common cause of mortality (3). However, the therapeutic options for HCC remain limited (4-6). Currently, chemotherapy is the most frequently used treatment for liver and other types of cancer. However, the toxicity of chemotherapeutic medicines in healthy tissues and cells remains a significant obstacle preventing the successful treatment of cancer with chemotherapy. There is, therefore, a requirement to identify novel therapeutic agents for hepatoma. Plants are a source of phytochemical compounds and secondary metabolites, which may have medicinal properties. Almost $60 \%$ of the drug treatments currently used are derivatives of naturally occurring compounds. The use of plants with medicinal properties in the treatment of cancer has increased due to their availability, low economic cost and relative lack of side effect, compared with commercially manufactured chemotherapeutic agents. As effective therapeutic strategies are required to combat diseases such as cancer, medicinal plants are good candidates due to their low toxicity profile in normal cells, whilst fighting different types of cancer (7).

Apoptosis or programmed cell death is an important process in the cytotoxicity induced by anticancer agents. The induction of apoptosis is associated with characteristic morphological and biochemical changes, which are facilitated by a series of gene regulatory cell-signaling pathways. Previously, perturbation of mitochondrial function has been observed to be required in the apoptotic cascade. Anticancer drugs may damage the mitochondria by increasing the permeability of the outer mitochondrial membrane, which is concomitant with breakdown of the mitochondrial membrane potential $(\Delta \Psi \mathrm{m})$, 
as a drop in $\Delta \Psi \mathrm{m}$ disturbs intracellular ATP synthesis, the production of reactive oxygen species, the mitochondrial redox ratio, the translocation of cytochrome $c$ to the cytosol and the degradation of caspase-3/poly ADP ribose polymerase. The mitochondria are hypothesized to be required for the induction of apoptosis, as changes occur within the mitochondria early during the apoptotic process (8-12). Previous studies have provided evidence that $\Delta \Psi \mathrm{m}$ is involved in the regulation of apoptosis within a cell. When apoptosis is triggered in response to specific physiological signals, a proteolytic cascade, involving a number of caspases, is initiated in the cell undergoing apoptosis, which leads to the activation of nucleases, thereby initiating the degradation of chromosomal DNA. This type of DNA fragmentation is a hallmark of the apoptotic process. A family of proteases, termed caspases, are activated in cells undergoing apoptosis. This results in the onset of numerous molecular and structural changes, including condensation of nuclear heterochromatin, cell shrinkage, loss of the positional organization of the cell organelles in the cytoplasm and degradation of DNA repair enzymes $(13,14)$.

Given the limited therapeutic options available for HCC, the present study was undertaken in order to evaluate the anticancer activity of oleanolic acid, a plant based triterpene, in the HepG2 human HCC cell line. In addition the study aimed to investigate the underlying mechanism of action of oleanolic acid, by evaluating its effects on apoptosis, using staining methods in order to analyze cell cycle phase distribution and changes in $\Delta \Psi \mathrm{m}$, as well as examining its effects on DNA fragmentation using gel electrophoresis.

\section{Materials and methods}

Chemicals and biochemicals. Oleanolic acid was obtained from Sigma-Aldrich (St. Louis, MO, USA) and $100 \mathrm{mg} / \mathrm{ml}$ solution, dissolved in dimethyl sulfoxide (DMSO), was stored at $-20^{\circ} \mathrm{C}$ prior to use. RPMI 1640 medium, fetal bovine serum (FBS) and penicillin-streptomycin were obtained from Hangzhou Sijiqing Biological Engineering Materials Co., Ltd. (Hangzhou, China). An MTT kit was obtained from Roche Molecular Biochemicals (Indianapolis, IN, USA). The Annexin V-fluorescein isothiocyanate (FITC)-propidium iodide apoptosis detection kit and acridine orange (AO) dye was obtained from Sigma-Aldrich. All other chemicals and solvents used were of the highest purity grade. Cell culture plasticware was obtained from BD Falcon (Franklin Lakes, NJ, USA).

Cell lines. The HepG2 human HCC cell line was obtained from the Shanghai Institute of Cell Resource Center of Life Science (Shanghai, China). The cells were maintained in RPMI-1640 supplemented with $10 \%$ FBS with penicillin $(100 \mathrm{U} / \mathrm{ml})$ and streptomycin $(100 \mu \mathrm{g} / \mathrm{ml})$ in a humidified atmosphere of $50 \mu \mathrm{g} / \mathrm{ml} \mathrm{CO}_{2}$ at $37^{\circ} \mathrm{C}$.

Cell viability assay. The association between HepG2 cell growth inhibition and oleanolic acid concentration was investigated using an MTT assay. Briefly, the cells were placed within 96-well culture plates $\left(3 \times 10^{6}\right.$ cells/well). After $24 \mathrm{~h}$ adherence, the cells were treated with 5,25 or $50 \mu \mathrm{M}$ oleanolic acid, or $0.1 \%$ DMSO for between 0 and $96 \mathrm{~h}$. The medium was replaced at 2-day intervals. At the end of the treatment, MTT
$(10 \mathrm{mg} / \mathrm{ml})$ was added to each well. The cells were incubated for a further $4 \mathrm{~h}$ and $150 \mu \mathrm{l}$ DMSO was then added to each well. Absorbance at $570 \mathrm{~nm}$ was measured and the growth inhibition ratio was calculated. A total of three independent experiments were performed. The half-maximal inhibitory concentration values were obtained from the MTT viability curves using GraphPad Prism 4.0 software (GraphPad Software,. Inc., San Diego, CA, USA).

Fluorescence microscopy examination of apoptosis. HepG2 cells were plated in six-well plates (Guangzhou Jet Bio-Filtration Products Co., Ltd., Guangzhou, China) at a density of $1 \times 10^{5}$ cells $/ \mathrm{ml}$ and then cultured for $24 \mathrm{~h}$ to allow complete attachment to the surface of the plates. Subsequently, the cells were treated with various concentrations of oleanolic acid treatment $(0,5,25$ and $50 \mu \mathrm{M})$ for $24 \mathrm{~h}$. Following oleanolic acid treatment, the culture plates were observed using an inverted light microscope (Nikon Instruments Eclipse Ti-E; Nikon, Sendai, Japan). For the other cells, a staining method using AO and ethidium bromide (EB; Sigma-Aldrich) was performed following incubation. HepG2 cells were treated with yarious concentrations of oleanolic acid $(0,5,25,50 \mu \mathrm{M})$ for $72 \mathrm{~h}$. Subsequently, cells on coverslips were collected, washed with phosphate-buffered saline (PBS) twice, stained with $\mathrm{AO} / \mathrm{EB}$ solution $(20 \mu \mathrm{g} / \mathrm{ml})$ and images were then captured using a fluorescence microscope (Nikon, Tokyo, Japan).

Effect of oleanolic acid on cell cycle phase distribution. Progress through the cell cycle was analyzed using a FACSCalibur instrument (BD Biosciences, San Jose, CA, USA), equipped with CellQuest 3.3 software. ModFit LT cell cycle analysis software (Modfit LT 2.0; Verity Software House Inc.; Topsham, ME, USA) was used to determine the percentage of cells in the different phases of the cell cycle.

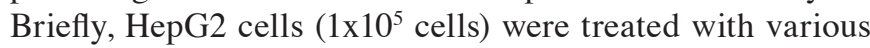
concentrations of oleanolic acid $(0,5,25$ and $50 \mu \mathrm{M})$ for $48 \mathrm{~h}$. Subsequently, the cells were collected, washed with ice cold PBS, fixed with $70 \%$ alcohol at $4^{\circ} \mathrm{C}$ for $12 \mathrm{~h}$ and stained with propidium iodide in the presence of $1 \%$ RNAase $\mathrm{A}$ at $37^{\circ} \mathrm{C}$ for 30 min prior to analysis using flow cytometry (BD Biosciences, San Jose, CA, USA).

Quantification of apoptotic cell death. Apoptosis was assayed using an Annexin V-FITC apoptosis detection kit (Calbiochem, Darmstadt, Germany). Cells treated with or without oleanolic acid were stained with propidium iodide and Annexin V-FITC, according to the manufacturer's instructions. The percentage of live, apoptotic and necrotic cells were analyzed by flow cytometry (BD Biosciences). Data from $10^{5}$ cells were analyzed for each sample. Spectrophotometry at $405 \mathrm{~nm}$ using ELISA based cell death detection kits (Calbiochem) was employed to detect apoptotic cell death by measuring the level of DNA fragmentation in the lysates of cells, which were treated or untreated with andrographolide and its analogues. Andrographolide is a diterpenoid lactone, which reduces the DNA binding of nuclear factor- $\kappa \mathrm{B}$ in whole cells.

DNA fragmentation assay. HepG2 HCC cells were seeded in a 100-mm cell culture dish for $24 \mathrm{~h}$, and treated with 5, 25 and $25 \mu \mathrm{M}$ oleanolic acid for $72 \mathrm{~h}$. The control and treated cells were 
$\mathbf{A}$

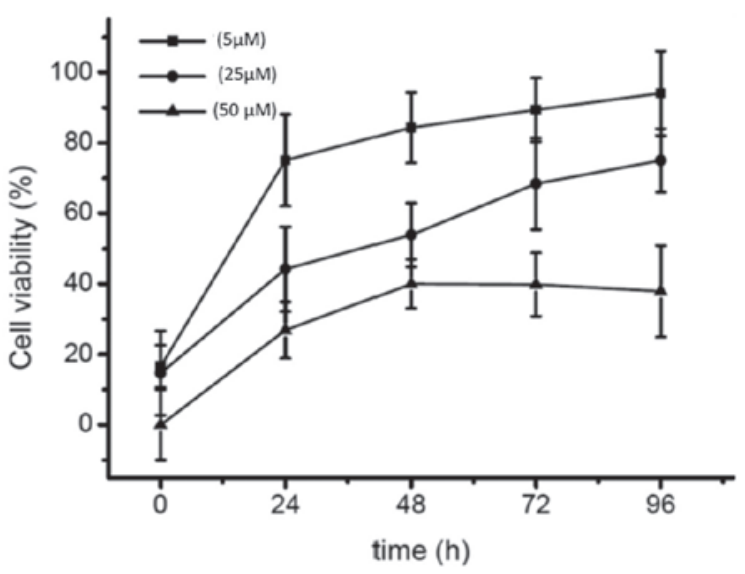

B

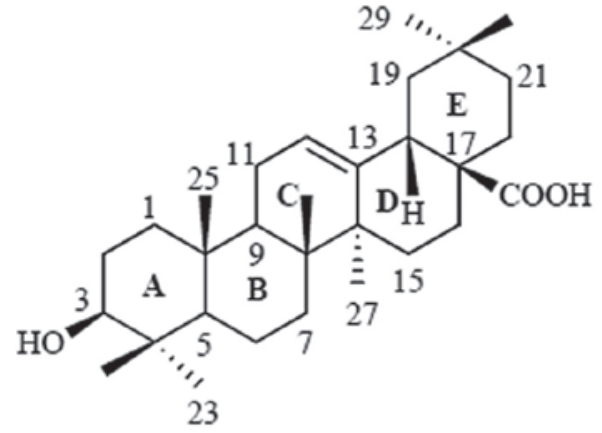

oleanolic acid

Figure 1. (A) Cytotoxic effect of oleanolic acid on HepG2 cells at various concentrations and time intervals. (B) Chemical structure of oleanolic acid.

harvested and washed with PBS, and the pellets were lysed with a $200 \mu \mathrm{l}$ DNA lysis buffer (1\% NP-40, $10 \mathrm{mM}$ EDTA, $50 \mathrm{mM}$ Tris- $\mathrm{HCl}$ ) for $20 \mathrm{~min}$. Following centrifugation at $1,568 \mathrm{x}$ f for $15 \mathrm{~min}$, the supernatants were prepared in an equal volume of $1.5 \%$ sodium-dodecyl sulphate, incubated with $5 \mathrm{mg} / \mathrm{ml} \mathrm{RNase} \mathrm{A}$ at $60^{\circ} \mathrm{C}$ for $2 \mathrm{~h}$ followed by digestion with $2.5 \mathrm{mg} / \mathrm{ml}$ proteinase $\mathrm{K}$ for $2 \mathrm{~h}$ at $20^{\circ} \mathrm{C}$. Following the addition of 0.5 volumes of $10 \mathrm{M}$ ammonium acetate, the DNA was precipitated with 2.5 volumes of cold ethanol and collected by centrifugation at $1,680 \mathrm{x}$ g for $30 \mathrm{~min}$. It was then dissolved in gel loading buffer, separated by electrophoresís in $1.5 \%$ agarose gel and visualized under UV light, following EB staining.

$\Delta \Psi$ m loss in HepG2 cells. $\Delta \Psi \mathrm{m}$ in Hep 2 cells was measured using rhodamine-123 dye (Sigma-Aldrich). HepG2 cells $\left(5 \times 10^{6}\right)$ were treated with various concentrations $(0,5,25$ and $50 \mu \mathrm{M})$ of oleanolic acid and $\Delta \Psi \mathrm{m}$ was then measured using flow cytometry. Rhodamine-123 (5 mM) was added $2 \mathrm{~h}$ prior to the termination of the experiment. Subsequently, the cells were washed with PBS and incubated with propidium iodide $(10 \mu \mathrm{g} / \mathrm{ml})$ for $30 \mathrm{~min}$. Cells were then analyzed with a flow cytometer.

Statistical analysis. All data were analyzed using analysis of variance, followed by Dunnett's test for pairwise comparisons. Data are presented as the mean \pm standard deviation. Statistical analyses were performed using Graph Pad 5.0 (GraphPad Software, Inc., La Jolla, CA, USA). P $<0.05$ was considered to indicate a statistically significant difference.

\section{Results}

Oleanolic acid induces potent cytotoxic effects against Hep G2 cells. HepG2 cells were treated with various concentrations (5, 25 and $50 \mu \mathrm{M}$ ) of oleanolic acid for $24,48,72$ and $96 \mathrm{~h}$ and cell viability was then evaluated using an MTT assay. As shown in Fig. 1, oleanolic acid treatment resulted in a dose-dependent as well as a time-dependent reduction in cell viability. The percentage of growth inhibition at various concentrations in HCC cells was determined as the percentage of viable treated cells compared with viable untreated control cells.
Effect of oleanolic acid on cell cycle phase distribution. In order to demonstrate whether oleanolic acid induces cell cycle disturbances in HepG2 cells, flow cytometric analysis using propidium iodide as a staining agent was performed, following oleanolic acid treatment at various concentrations $(5,25$ and $50 \mu \mathrm{M}$ ) for $24 \mathrm{~h}$. As shown in Fig. 2, following treatment with oleanolic acid at $0,5,25$ and $50 \mu \mathrm{M}$ concentrations for $24 \mathrm{~h}$, significant G0/G1 cell cycle growth arrest was observed, while the number of cells in the $\mathrm{S}$ and G2-M phases was markedly reduced. At a low concentration of oleanolic acid, apoptosis was not observed. However, at 25 and $50 \mu \mathrm{M}$ concentrations, the fraction of cells undergoing apoptosis increased significantly up to 31.7 and $54.2 \%$ compared with untreated cells at $4.5 \%$.

Apoptosis induction in HepG2 cells as revealed by Annexin V-FITC analysis. An essential feature of apoptosis is the flipping of phosphatidyl serine (PS) from the inner surface to outer surface of the plasma membrane of the cells. PS is a phospholipid component usually positioned on the cytoplasmic surface of the cell membrane in viable normal cells. When an apoptotic event is induced in a cell, PS is no longer restricted to the cytosolic region of the membrane, but is exposed on the cell surface. As such, PS translocation is considered to be a biochemical marker of apoptosis. Annexin V staining is able to detect PS and may therefore be used for apoptosis analysis. When cells are stained with Annexin V in tandem with propidium iodide, this reagent enters the cell only once the plasma cell membrane has deteriorated. In the current study, flow cytometric analysis revealed that a higher number of Annexin V-positive cells were present in the oleanolic acid-treated HepG2 cells than in the control group (Figs. 3 and 4). The percentage of viable cells was low in the samples treated with lower concentration of oleanolic acid. However, at higher doses (25 and $50 \mu \mathrm{M})$, the total number of apoptotic cells significantly increased. The present study confirmed that oleanolic acid induces apoptosis in $\mathrm{HepG} 2$ cells.

Apoptotic morphological changes in HepG2 cells following oleanolic acid treatment. Apoptosis is a highly organized biochemical process, which eradicates injured or abnormal cells in multicellular organisms. In order to establish whether cell death induced by oleanolic acid is mediated through apoptosis, 
A

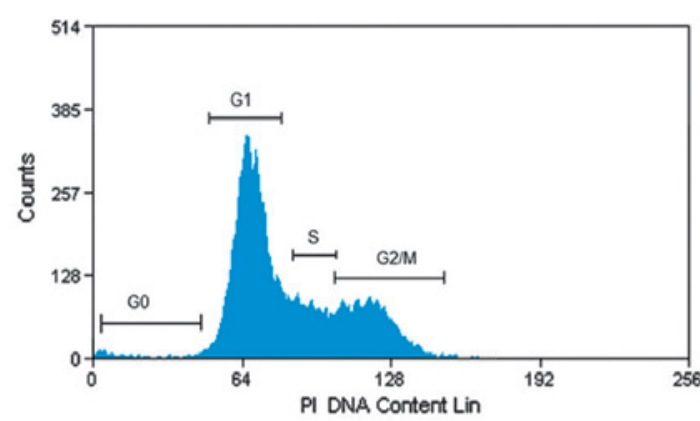

C

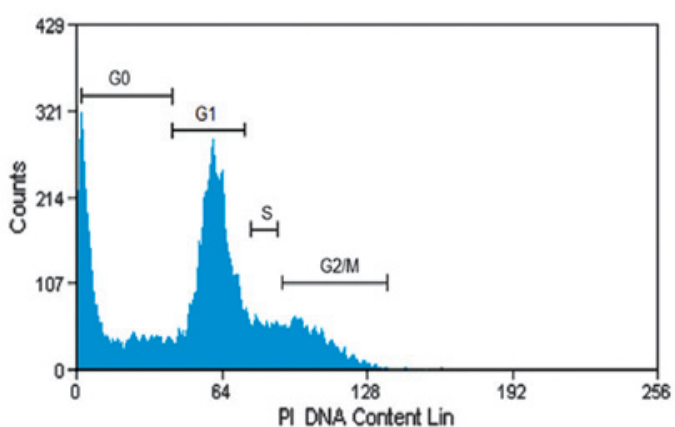

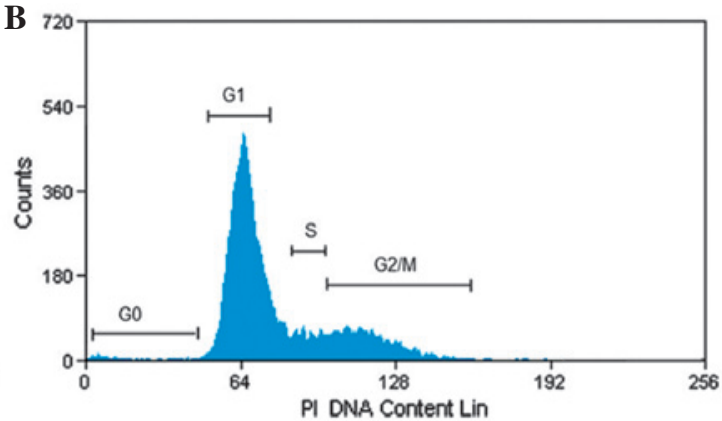

D

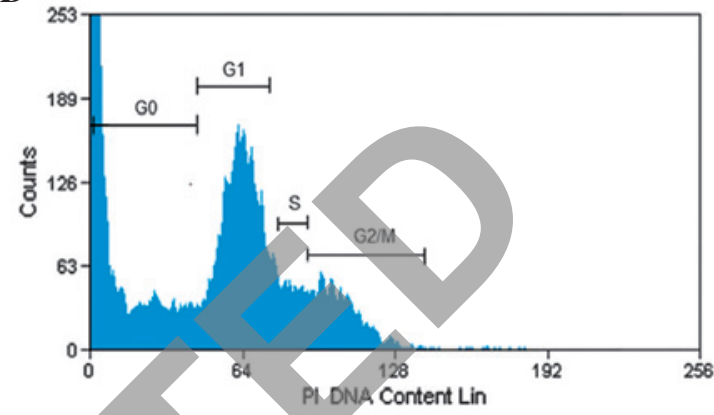

Figure 2. Cell cycle analysis of HepG2 cancer cells treated with oleanolic acid. The distribution of cells undergoing apoptosis and in various phases of the cell cycle was determined in HepG2 cells following treatment with oleanolic acid at various concentrations. (A) G0/G1-5\%, S-30\%, G2M-65\%; (B) G0/G1-15\%, S-25\% G2M-60\%; (C) G0/G1-38\%, S-20\%, G2M-42\%; (D) G0/G1-55\%, S-15\%, G2M-30\%; representing treatment with 0, 5,25 and $50 \mu \mathrm{M}$, respectively. Values are presented as the mean \pm standard error of mean of three determinations. PI, propidium iodide.
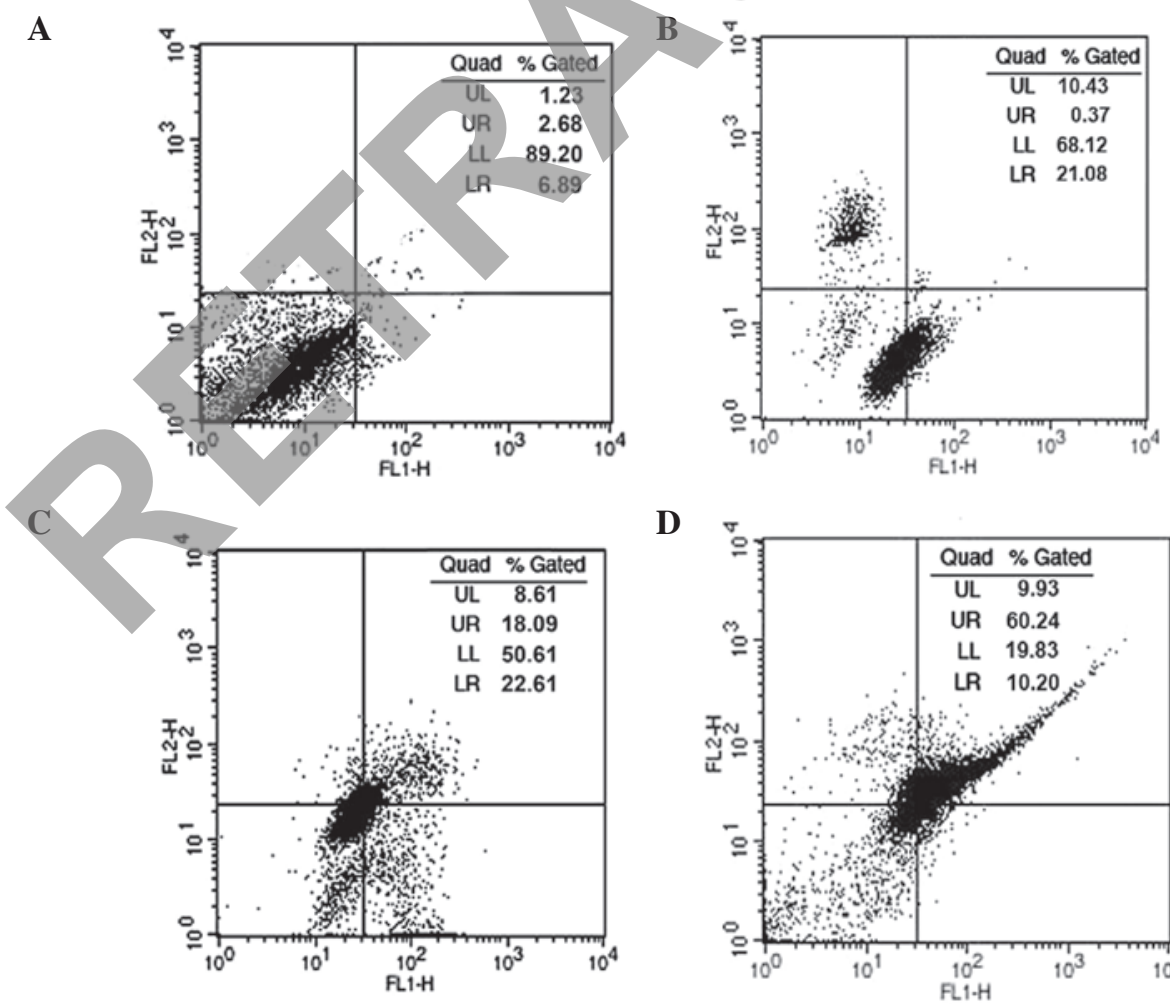

$\mathbf{D}$

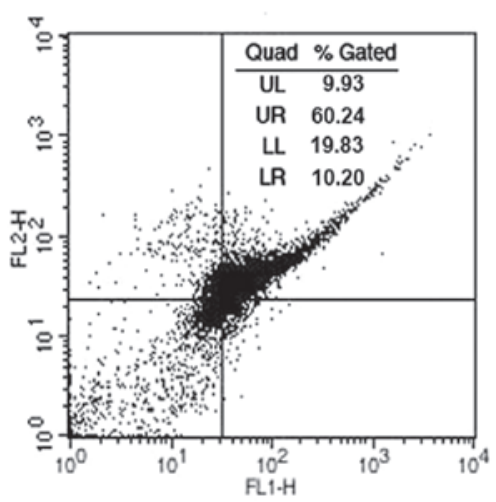

Figure 3. Analysis of apoptosis using flow cytometry in HepG2 cells. Cells were treated with various concentrations of oleanolic acid. (A-D) represent treatment with $0,5,25$ and $50 \mu \mathrm{M}$ oleanolic acid, respectively, for $48 \mathrm{~h}$. Binding of Annexin V/fluorescein isothiocyanate to phosphatidyl serine was determined by flow cytometry. The percentage of apoptotic cells determined by the number of Annexin V/propidium iodide-positive cells is shown in the scattered plot.

HepG2 cells were treated with several concentrations of oleanolic acid $(0,5,25$ and $50 \mu \mathrm{M})$ for $48 \mathrm{~h}$, and the characteristic morphological features of apoptosis were examined under an inverted light fluorescence microscope. As shown in Fig. 5, compared with viable cells, oleanolic acid treatment at 5 and $25 \mu \mathrm{M}$ resulted in the appearance of cell shrinkage along with 
A

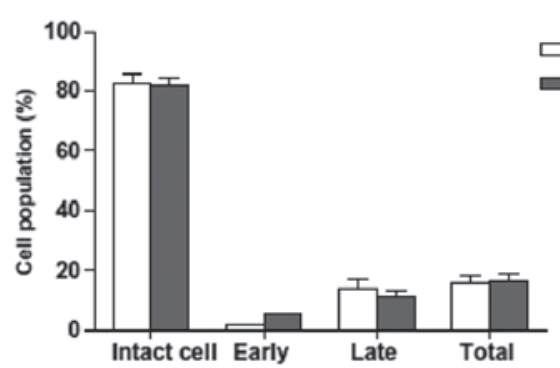

C

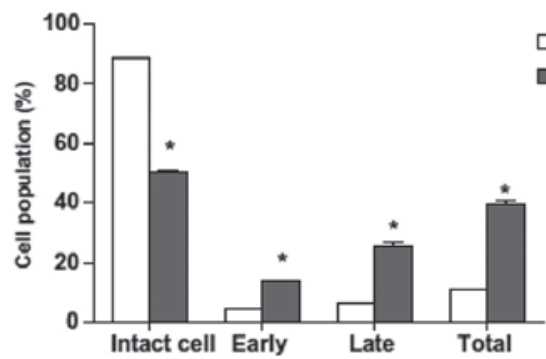

B

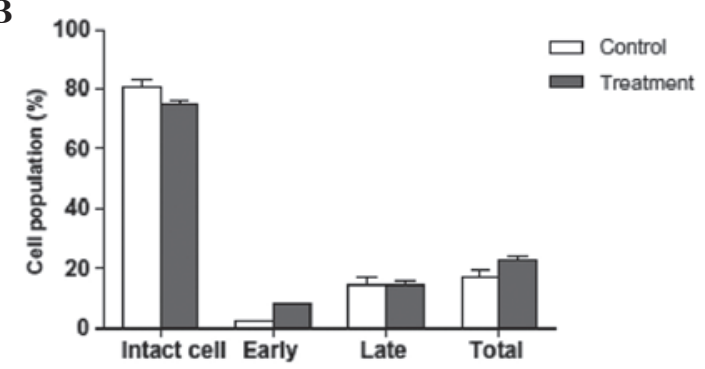

D

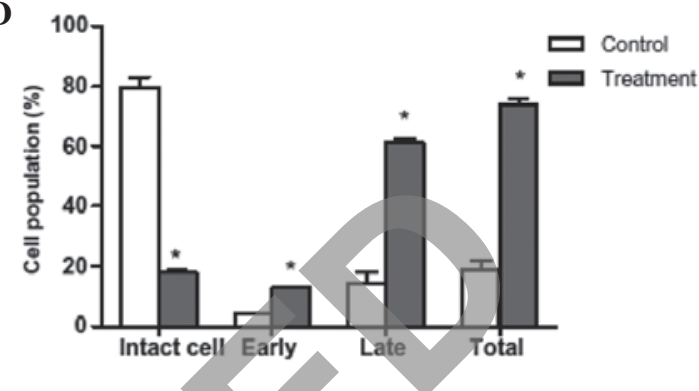

Figure 4. Apoptotic analysis of HepG2 cells treated with oleanolic acid at various concentrations. (A-D) represent treatment with $0,5,25$ and $50 \mu \mathrm{M}$ oleanolic acid, respectively. The distribution of cells undergoing early and late apoptosis, together with cells not undergoing apoptosis (intact cell) and the total extent of apoptosis, was determined in HepG2 cells, following treatment with oleanolic acid, using Annexin V/fluorescein isothiocyanate and propidium iodide flow cytometric analysis. ${ }^{*} \mathrm{P}<0.05$, compared with control.
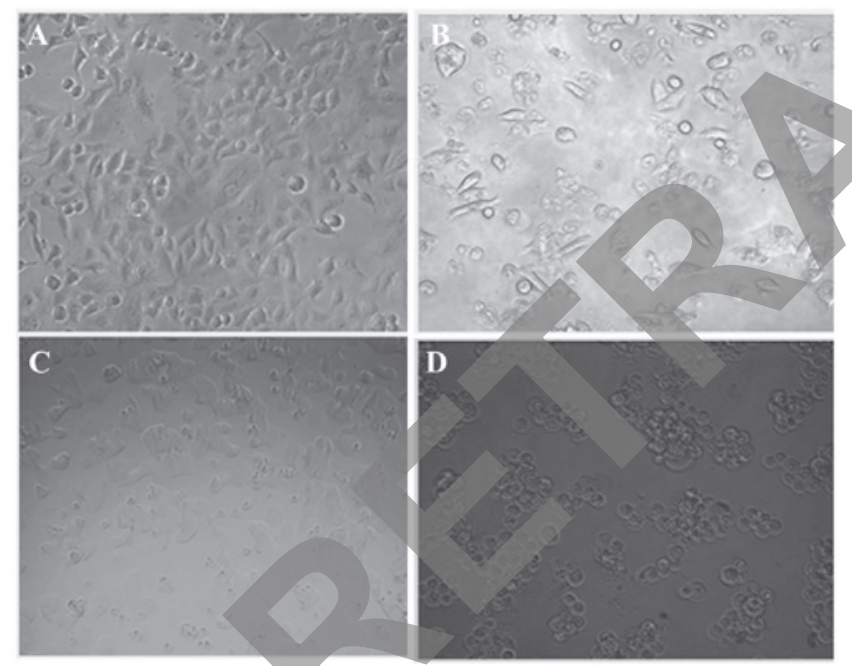

Figure 5. Analysis of morphological changes in oleanolic acid-treated HepG2 tumor cells. (A) Untreated HepG2 control cells. (B-D) HepG2 cells treated with 5,25 and $50 \mu \mathrm{M}$ oleanolic acid, respectively. Cells were exposed to various concentrations of oleanolic acid and morphological changes were observed following $48 \mathrm{~h}$ of treatment. Images were captured using an inverted microscope (magnification, $\mathrm{x} 20$ ).

membrane blebbing, which are characteristic features of cell apoptosis. When treated with $50 \mu \mathrm{M}$ oleanolic acid, almost all the HepG2 cancer cells shrank considerably and no cells with normal morphological features were observed.

Furthermore, $\mathrm{AO}$ and $\mathrm{EB}$ double staining was conducted in the HepG2 cells in order to observe cell apoptosis, with the assistance of a fluorescence microscope. Following staining with a mixture of $\mathrm{AO}$ and $\mathrm{EB}$, viable cells $(0 \mu \mathrm{M}$; Fig. $6 \mathrm{~A})$ were observed to have large green nuclei, indicating that their cell membranes had remained intact. However, when treated with 5 or $25 \mu \mathrm{M}$ of oleanolic acid, the number of cells with large green nuclei reduced significantly (Fig. 6). Furthermore,
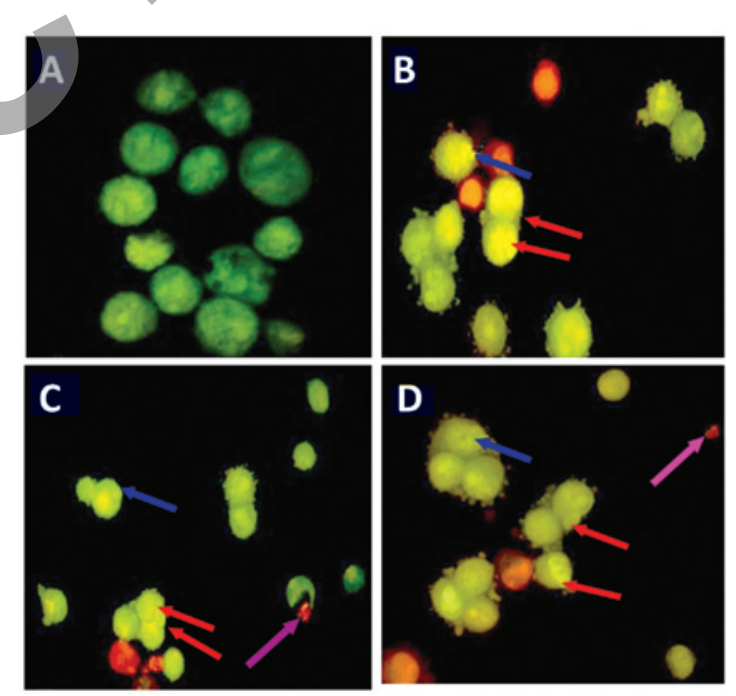

Figure 6. Morphological observation following acridine orange and ethidium bromide staining (magnification, $\mathrm{x} 400$ ). HepG2 cells were treated (A) without and (B) with oleanolic acid at $5 \mu \mathrm{M}$, (C) $25 \mu \mathrm{M}$ and (D) $50 \mu \mathrm{M}$ for $48 \mathrm{~h}$. Arrows indicate nuclei size and membrane integrity.

at a concentration of $50 \mu \mathrm{M}$, almost all cells exhibited signs of nuclear condensation and apoptotic body formation.

DNA fragmentation induced by oleanolic acid treatment. DNA fragmentation is a process, which damages DNA, leading to cell death that occurs via the activation of certain intrinsic agents, such as caspase 3 and 9. This cleavage produces ladders of DNA fragments that are the size of integer multiples of a nucleosome length (180-200 bp). The DNA fragmentation is initiated by caspase- 3 activation of inactive caspase-activated deoxyribonuclease (CAD), through removal of its inhibitors, such as inhibitor of CAD (ICAD) (9). As a biochemical hallmark of intrinsic apoptotic cell death, DNA 


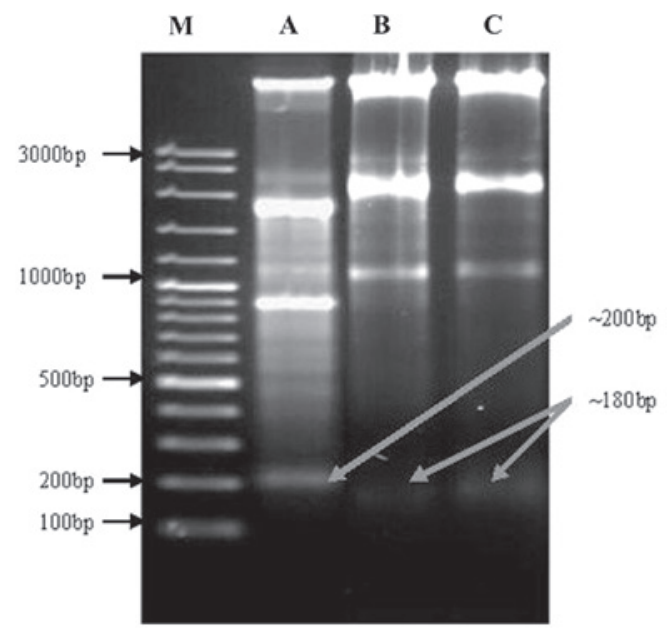

Figure 7. Gel electrophoresis of DNA samples isolated from HepG2 cancer cells following treatment with oleanolic acid. Cells were incubated with various concentrations of oleanolic acid for $72 \mathrm{~h}$. M, 100 bp DNA ladder marker; lane A, HepG2 cells treated with $5 \mu \mathrm{M}$ of oleanolic acid; lane B, HepG2 cells treated with $25 \mu \mathrm{M}$ of oleanolic acid; and lane C, HepG2 cells treated with $50 \mu \mathrm{M}$ of oleanolic acid. Each experiment was conducted in triplicate. DNA fragments were separated using 1.5\% agarose gel electrophoresis and visualized under UV light following staining with ethidium bromide.

fragmentation was used to determine whether the anticancer effect of oleanolic acid on cells occurs via the activation of caspases, mainly caspase-3. As shown in Fig. 7, marked DNA fragmentation was observed in HepG2 cancer cells following treatment with 5,25 and $50 \mu \mathrm{M}$ treatments of oleanolic acid for $72 \mathrm{~h}$. However, the control cells did not exhibit evident DNA laddering (data not shown). The treatment of HepG2 cells with oleanolic acid resulted in the induction of intrinsic apoptotic effects as low as $5 \mu \mathrm{M}$. Higher concentrations of oleanolic acid (lane 1-3; Fig. 7) for $72 \mathrm{~h}$ resulted in the presence of the typical features of DNA laddering on an agarose gel.

Oleanolic acid induced $\Delta \Psi m$ loss in HepG2 cells. An important and indicative stage in the intrinsic apoptotic pathway is the depolarization of the mitochondrial membrane and the subsequent increase in permeability of the outer membrane, following pore formation. This is accompanied by the release of proapoptotic molecules and cytochrome $c$. The fluorescent dye, rhodamine-123, is a specific probe for the detection of alterations in $\Delta \Psi \mathrm{m}$ in living cells. The present results demonstrated that oleanolic acid induced a significant reduction in the number of cells with an intact membrane potential and increased the number of cells with low $\Delta \Psi \mathrm{m}$ after $48 \mathrm{~h}$. Loss of $\Delta \Psi \mathrm{m}$ is an essential event in the mitochondrial pathway of apoptosis. The $\Delta \Psi \mathrm{m}$ level was observed to be reduced following treatment with increasing concentrations of oleanolic acid in HepG2 cells over $48 \mathrm{~h}$ (Fig. 8A and B). Disruption of $\Delta \Psi \mathrm{m}$ was relatively low in untreated cells.

\section{Discussion}

$\mathrm{HCC}$ is an aggressive form of cancer, with high rates of morbidity and mortality, a poor prognosis and limited therapeutic options. Despite evidence that chemotherapy is one of the most effective therapeutic approaches for HCC, the

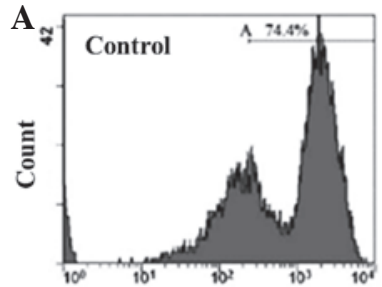

Flourescence
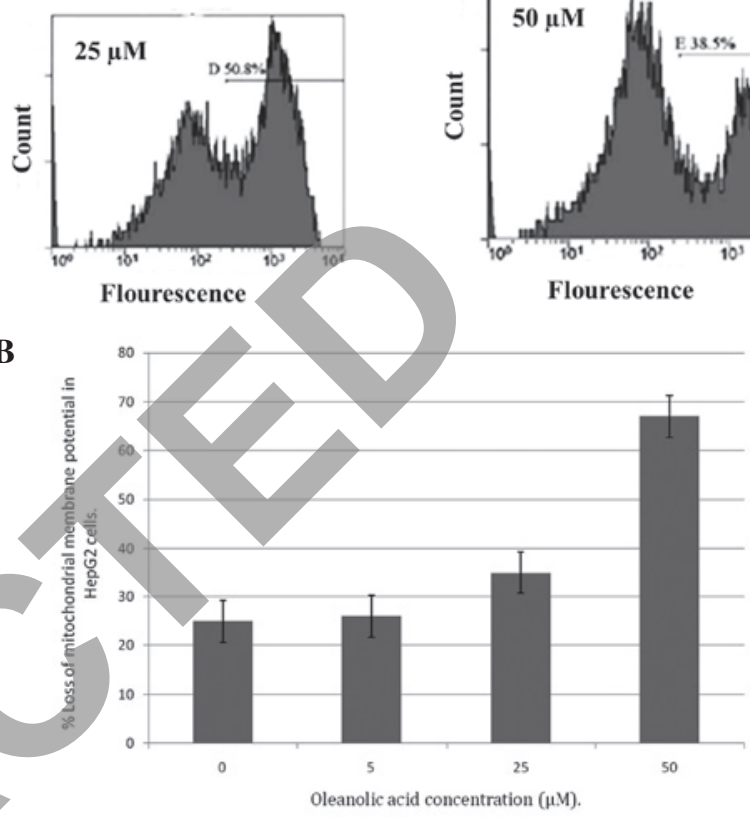

Figure 8. (A) Effect of oleanolic acid on the mitochondrial $\Delta \Psi \mathrm{m}$ of HepG2 cells. Cells treated with oleanolic acid for $48 \mathrm{~h}$ were incubated with rhodamine-123 and measured using flow cytometry. The percentages of cells in the right section of the histogram indicate the number of $\Delta \Psi \mathrm{m}$-collapsed cells. (B) Percentage loss of $\Delta \Psi \mathrm{m}$ in the oleanolic acid-treated cells. $\Delta \Psi \mathrm{m}$, membrane potential.

toxic side-effects associated with it are difficult for patients to tolerate. Therefore, there is a requirement for the development of novel and effective drugs, which are more efficacious, yet at the same time produce fewer serious side-effects. Therapeutics based on natural products, such as the use of plant-derived natural products and Traditional Chinese Medicine in cancer treatment, may minimise adverse drug effects. Medicinal and aromatic plants are a rich source of compounds with anticancer properties and produce low toxicity in normal cells. Therefore, increasing attention has been placed on identifying novel anticancer drug treatments from natural sources (15-17).

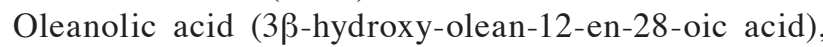
an oleanane triterpenoid, is a ubiquitous triterpenoid in the plant kingdom and is integral part of the human diet (18). A number of published studies have revealed that oleanolic acid possesses important pharmacological properties. Oleanolic acid has been reported to inhibit tumor promotion, induced by 12-O-tetradecanoyl-phorbol-13-acetate, in vivo. It was able to effectively inhibit the promotion of tumorigenesis in the skin of mice (19-21). The cytotoxic effect of oleanolic acid on the jurkat cell line ( $\mathrm{T}$ cell lymphoma) has also been previously demonstrated (22). The antitumoral mechanism of oleanolic 
acid is hypothesized to be mediated via the killing of cells with cytotoxin at a high dose, and the inhibition of cell proliferation at a low dose. In other studies, oleanolic acid derivatives isolated from the aerial parts of Ficus microcarpa were observed to exert cytotoxic activities in vivo against three human cancer cell lines; namely, HONE-1 nasopharyngeal carcinoma, KB oral epidermoid carcinoma and HT29 colorectal carcinoma cells (23). Furthermore, oleanolic acid has also been reported to inhibit the proliferation of K562 human erythroleukemia cells (23).

Although previous studies have revealed that oleanolic acid exhibits anticancer activities against a wide range of malignancies, the mechanism of action of oleanolic acid in cancer cells has not been investigated in detail. The objective of the present study was to determine the mechanism underlying the anticancer action of oleanolic acid in HepG2 HCC cells by evaluating its effects on cell viability, cell cycle phase distribution, apoptosis, DNA fragmentation and $\Delta \Psi \mathrm{m}$. To the best of our knowledge, the current study is the first of this nature.

In conclusion, the present results revealed that oleanolic acid produces potent growth inhibition of HepG2 hepatocellular cancer cells in vitro and showed that this effect is mediated through arrest of the cell cycle, the induction of apoptosis and DNA fragmentation, and a loss of $\Delta \Psi \mathrm{m}$ in cancer cells. Therefore, oleanolic acid has the potential to be developed further as an anticancer agent in the treatment of HCC.

\section{References}

1. Jemal A , Bray F, Center MM, Ferlay J, Ward E and Forman D: Global cancer statistics. CA Cancer J Clin 61: 69-90, 2011.

2. El-Serag HB and Rudolph KL: Hepatocellular carcinoma: epidemiology and molecular carcinogenesis. Gastroenterology 132: 2557-2576, 2007.

3. Ananthakrishnan A, Gogineni V and Saeian K: Epidemiology of primary and secondary liver cancers. Semin Intervent Radiol 23: 47-63, 2006.

4. McGlynn KA, Tsao L, Hsing AW, Devesa SS and Fraumeni JF Jr: International trends and patterns of primary liver cancer. Int J Cancer 94: 290-296, 2001.

5. Röcken C and Carl-McGrath S: Pathology and pathogenesis of hepatocellular carcinoma. Dig Dis 19: 269-278, 2001.

6. Kaufmann SH and Earnshaw WC: Induction of apoptosis by cancer chemotherapy. Exp Cell Res 256: 42-49, 2000.
7. Pezzuto JM: Plant-derived anticancer agents. Biochem Pharmacol 53: 121-133, 1997.

8. Ashkenazi A and Dixit VM: Death receptors: signaling and modulation. Science 281: 1305-1308, 1998.

9. Green DR and Reed JC: Mitochondria and apoptosis. Science 281: 1309-1312, 1998.

10. Okada H and Mak TW: Pathways of apoptotic and non-apoptotic death in tumour cells. Nat Rev Cancer 4: 592-603, 2004.

11. Philchenkov A: Caspases: potential targets for regulating cell death. J Cell Mol Med 8: 432-444, 2004.

12. Sharifi AM, Eslami H, Larijani B and Davoodi J: Involvement of caspase-8, -9 and -3 in high glucose-induced apoptosis in PC12 cells. Neurosci Lett 459: 47-51, 2009.

13. Kluck RM, Bossy-Wetzel E, Green DR and Newmeyer DD: The release of cytochrome c from mitochondria: a primary site for Bcl-2 regulation of apoptosis. Science 275: 1132-1136, 1997.

14. Narita M, Shimizu S, Ito T, Chittenden T, et al: Bax interacts with the permeability transition pore to induce permeability transition and cytochrome $c$ release in isolated mitochondria. Proc Natl Acad Sci USA 95: 14681-14686, 1998.

15. Mukherjee AK, Basu S, Sarkar N and Ghosh AC: Advances in cancer therapy with plant based natural products. Curr Med Chem 8: 1467-1486, 2001

16. Wang S, Penchala S, Prabhu S, Wang J and Huang Y: Molecular basis of traditional Chinese medicine in cancer chemoprevention. Curr Drug Discov Technol 7: 67-75, 2010.

17. Desai AG, Qazi GN, Ganju RK,El-Tame M, et al: Medicinal plants and cancer chemoprevention. Curr Drug Metab 9: 581-591, 2008.

18. Somova LO, Nadar A, Rammanan P and Shode FO: Cardiovascular, antihyperlipidemic and antioxidant effects of oleanolic and ursolic acids in experimental hypertension. Phytomedicine 10: 115-121, 2003.

19. Ohigashi H, Takamura H, Koshimizu K, Tokuda $\mathrm{H}$ and Ito $\mathrm{Y}$ : Search for possible antitumor promoters by inhibition of 12-O-tetradecanoylphorbol-13-acetate-induced Epstein-Barr virus activation; ursolic acid and oleanolic acid from an anti-inflammatory Chinese medicinal plant, Glechoma hederaceae L. Cancer Lett 30: 143-151, 1986.

20. Tokuda H, Ohigashi H, Koshimizu K and Ito Y: Inhibitory effects of ursolic and oleanolic acid on skin tumor promotion by 12-O-tetradecanoylphorbol-13-acetate. Cancer Lett 33: 279-285, 1986.

21. Huang D, Ding Y, Li Y, Zhang W, et al: Anti-tumor activity of a 3-oxo derivative of oleanolic acid. Cancer Lett 233: 289-296, 2006.

22. Li J, Xu LZ, Zhu WP, Zhang TM, Li XM, Jin AP, Huang KM, Li DL and Yang QY: Effects of ursolic acid and oleanolic acid on Jurkat lymphoma cell line in vitro. Zhongguo Aizheng Zazhi 9: 395-397, 1999.

23. Chiang YM, Chang JY, Kuo CC, Chang CY and Kuo YH: Cytotoxic triterpenes from the aerial roots of Ficus microcarpa. Phytochemistry 66: 495-501, 2005. 